\title{
Functional Representation of the Ablowitz-Ladik Hierarchy. II
}

\author{
V E VEKSLERCHIK \\ Departamento de Matemáticas, E. T. S. I. Industriales, \\ Universidad de Castilla-La Mancha, \\ Avenida de Camilo José Cela, 3, 13071 Ciudad Real, Spain \\ On leave from the Institute for Radiophysics and Electronics, Kharkov, Ukraine \\ Regular Associate of the Abdus Salam ICTP, Trieste, Italy
}

Received July 27, 2001; Revised October 31, 2001; Accepted December 17, 2001

\begin{abstract}
In this paper we continue studies of the functional representation of the AblowitzLadik hierarchy $(\mathrm{ALH})$. Using formal series solutions of the zero-curvature condition we rederive the functional equations for the tau-functions of the ALH and obtain some new equations which provide more straightforward description of the ALH and which were absent in our previous paper. These results are used to establish relations between the ALH and the discrete-time nonlinear Schrödinger equations, to deduce the superposition formulae (Fay's identities) for the tau-functions of the hierarchy and to obtain some new results related to the Lax representation of the ALH and its conservation laws. Using the previously found connections between the ALH and other integrable systems we derive functional equations which are equivalent to the AKNS, derivative nonlinear Schrödinger and Davey-Stewartson hierarchies.
\end{abstract}

\section{Introduction.}

In 11 we have presented some results on the functional representation of the AblowitzLadik hierarchy (ALH). The ALH, which had been originally proposed as an infinite set of the differential-difference equations (see [2]), has been written as a finite system of functional-difference equations. The aim of this work is to continue these studies. However, to present some formulae which were absent in [1] (such as, first of all, the functional relations written in terms of the solutions themselves, and not in terms of the corresponding tau-functions) is not the only (and not even the main) goal of this work. We wish to fill some gaps in the theory of the ALH. The ALH, which was discovered by Ablowitz and Ladik a quarter of century ago, is one of the first integrable discrete $(1+1)$-dimensional systems. Nevertheless, maybe because of lack of interesting practical applications, it has not received proper attention from mathematicians and physicists. In the not so distant past the 'classical' inverse scattering transform (IST) of [2] and Hirota's bilinear method, which were elaborated in the seventies, were almost the only tools for 
investigating the ALH. Other techniques developed during the last two decades (such as, e.g., formal series, Sato's approaches, discrete-time representation etc) have not been adopted to this integrable system. (Only recently a few works have appeared in which the ALH is considered from more modern viewpoints, see, e.g., [3, 俚.) The paper [1] was not an exception. The method of [1] is the method of the pioneering article [2]: the main equations of [1] were obtained by analyzing the structure of the Jost functions of the auxiliary scattering problem. The Jost functions, by definition, crucially depend on the boundary conditions imposed, while the form of the equations of the hierarchy, and hence the resulting functional equations, do not. Here we exploit another approach which is based on the local properties of the ALH and will enable us to avoid restrictions caused by the boundary conditions (the proof of main results in [1], but not the results themselves, was limited to the case of zero boundary conditions). This also gives us the possibility to see how the main objects of modern theory of integrability appear in the case of the ALH.

We start with the standard zero-curvature (or the so-called $U-V$ ) representation (ZCR) based on the $U$-matrix of the work [2], but instead of considering the polynomial, in auxiliary parameter $\lambda$, of $V$-matrices $V_{n}^{j}$ (as is in 'classical' IST, [2]) we deal with some formal matrix series from which the former can be obtained by multiplication plus projection scheme, $V_{n}^{j}(\lambda)=\pi_{\lambda}^{+}\left[\lambda^{-2 j} \mathcal{V}_{n}(\lambda)\right]$ (the definitions of $V_{n}^{j}(\lambda), \mathcal{V}_{n}(\lambda), \pi_{\lambda}^{ \pm}$as well as basic facts related to the ZCR one can find in Section 22). This approach is widely used in the theory of the $\mathrm{KdV}$, KP and other hierarchies but, to my knowledge, has not been elaborated in the case of the ALH. Then we show how the operator $\lambda d / d \lambda$, the importance of which has been discussed in the literature (see, e.g., [5]), acts on these series, which in a natural way leads to the so-called Miwa's shifts, $z_{k} \rightarrow z_{k}+\varepsilon \zeta^{k} / k$ [6]. The Miwa's shifts are one of the most characteristic attributes of the modern theory of integrability, which in such or another form are present in considerable part of recent works devoted to the KP (together with its modifications), KdV, 2D Toda, Davey-Stewartson and other hierarchies. However, in the context of the ALH they, to my knowledge, have not been discussed in the literature. After deriving the functional equations which are equivalent to the ALH (Section 3) and rewriting them in terms of the tau-functions of the ALH (Section 4 ) we address the question of the relations between these equations and the discrete-time Ablowitz-Ladik equations [7, 佨. Having established the discrete-time character of the functional representation of the ALH, i.e. considering the combined action of the evolutionary ALH flows as the integrable mapping of the work [4], it is rather natural to address the problem of superposition of these maps (i.e. superposition of the Miwa's shifts). In Section 6 we present Fay's formulae for the ALH which relate functions calculated at different values of arguments, $z_{k}+\sum_{a} \varepsilon_{a} \zeta_{a}^{k} / k$, and this in turn provides some interesting results related to the question of the higher-order discrete-time Ablowitz-Ladik equations [8] (see Section 7).

Thus in this paper we explicitly construct the chain from the scattering problem of Ablowitz and Ladik (written in 1975) through formal series and Miwa's shifts to the discrete-time equations written by Suris in 1997. However, it seems useful before we move forward (say, to the Grassmannian description of the ALH, which, again, is still to be developed) that we return to some 'classical' problems and demonstrate that the functional representation possesses some practical value. The key moment is that now we know the (formal) solution of the auxiliary linear problems, which enables us to obtain some new expressions for the results which have been formulated in terms of Jost functions and the 
scattering matrices. To this end we discuss once more the question of conservation laws. The form of the generating function for the divergent-like conservation laws presented in Section 8 seems to be new and rather convenient for practical usage.

Lastly we demonstrate that some of the ideas and results of this paper can be useful beyond the theory of the ALH. To do this we give in Section 9 few examples of the 'embedding into the ALH' approach: starting from the ALH functional equations we derive the functional representation of some other integrable models, namely, the derivative nonlinear Schrödinger (NLS), AKNS and Davey-Stewartson hierarchies.

\section{Zero curvature representation of the ALH}

The ALH is an infinite set of differential-difference equations, which can be presented as the compatibility condition for the linear system

$$
\begin{gathered}
\Psi_{n+1}=U_{n} \Psi_{n}, \\
\partial \Psi_{n}=V_{n} \Psi_{n},
\end{gathered}
$$

where $\Psi_{n}$ is a 2-column (or $2 \times 2$ matrix), $U_{n}$ and $V_{n}$ are $2 \times 2$ matrices with $U_{n}$ being given by

$$
U_{n}=U_{n}(\lambda)=\left(\begin{array}{cc}
\lambda & r_{n} \\
q_{n} & \lambda^{-1}
\end{array}\right)
$$

(here $\lambda$ is an auxiliary constant parameter). To provide the self-consistency of the system (2.1), (2.2) the matrices $V_{n}$ have to satisfy the following equation

$$
\partial U_{n}=V_{n+1} U_{n}-U_{n} V_{n}
$$

One can divide the ALH into two subsystems (subhierarchies): the 'positive' one (with the $V$-matrices being polynomials in $\lambda^{-1}$ ) and the 'negative' one (with the $V$-matrices being polynomials in $\lambda$ ). Since these subhierarchies are rather similar, we concentrate mostly on the 'positive' subsystem which stems from (2.1) and

$$
\partial_{j} \Psi_{n} \equiv \frac{\partial}{\partial z_{j}} \Psi_{n}=V_{n}^{j} \Psi_{n}, \quad j=1,2, \ldots
$$

where the matrices $V_{n}^{j}$ are polynomials of the $(2 j)$ th order in $\lambda^{-1}$ with the following structure:

$$
V_{n}^{j}=\lambda^{-2} V_{n}^{j-1}+\left(\begin{array}{cc}
\lambda^{-2} \alpha_{n}^{j} & \lambda^{-1} \beta_{n}^{j} \\
\lambda^{-1} \gamma_{n}^{j} & \delta_{n}^{j}
\end{array}\right), \quad j=2,3, \ldots
$$

Substituting (2.6) in the zero-curvature representation (2.4) one can obtain the set of equations determining the quantities $\alpha_{n}^{j}, \ldots, \delta_{n}^{j}$

$$
\begin{gathered}
\alpha_{n}^{j}=-\delta_{n}^{j-1}, \\
\beta_{n}^{j}=\beta_{n-1}^{j-1}+r_{n-1}\left(\delta_{n-1}^{j-1}+\delta_{n}^{j-1}\right), \\
\gamma_{n}^{j}=\gamma_{n+1}^{j-1}+q_{n}\left(\delta_{n}^{j-1}+\delta_{n+1}^{j-1}\right), \\
\delta_{n+1}^{j}-\delta_{n}^{j}=q_{n} \beta_{n}^{j}-r_{n} \gamma_{n+1}^{j}
\end{gathered}
$$


and present equations of the hierarchy as

$$
\partial_{j} q_{n}=q_{n} \delta_{n+1}^{j}+\gamma_{n+1}^{j}
$$

and

$$
\partial_{j} r_{n}=-r_{n} \delta_{n}^{j}-\beta_{n}^{j} .
$$

The $V$-matrices of the 'negative' subhierarchy, $\bar{V}_{n}$ (in this paper the overbar does not mean the complex conjugation!) possess the following structure:

$$
\bar{V}_{n}^{j}=\lambda^{2} \bar{V}_{n}^{j-1}+\left(\begin{array}{cc}
\bar{\alpha}_{n}^{j} & \lambda \bar{\beta}_{n}^{j} \\
\lambda \bar{\gamma}_{n}^{j} & \lambda^{2} \bar{\delta}_{n}^{j}
\end{array}\right)
$$

and the equations of the subhierarchy can be written as

$$
\bar{\partial}_{j} q_{n}=-q_{n} \bar{\alpha}_{n}^{j}-\bar{\gamma}_{n}^{j}
$$

and

$$
\bar{\partial}_{j} r_{n}=r_{n} \bar{\alpha}_{n+1}^{j}+\bar{\beta}_{n+1}^{j},
$$

where $\bar{\partial}_{j}=\partial / \partial \bar{z}_{j}$. All equations (2.11), (2.14) and (2.12), (2.15) are compatible. Thus we consider the $q_{n}$ and $r_{n}$ as functions of the infinite number of variables $z_{j}$ and $\bar{z}_{j}$ and write, e.g., $q_{n}(z, \bar{z})$ bearing in mind

$$
q_{n}(z, \bar{z})=q_{n}\left(z_{1}, \bar{z}_{1}, z_{2}, \bar{z}_{2}, \ldots\right) .
$$

Till now we were following the standard zero-curvature scheme of [2] (see also, e.g., [11]), but hereafter, since our purpose is to discuss the ALH as a whole, we deal not with the quantities $\alpha_{n}^{j}, \ldots, \delta_{n}^{j}$ (which describe the $j$ th flow) but with series defined by

$$
\left(\begin{array}{ll}
a_{n}(\zeta) & b_{n}(\zeta) \\
c_{n}(\zeta) & d_{n}(\zeta)
\end{array}\right)=\sum_{j=1}^{\infty} \zeta^{j}\left(\begin{array}{cc}
\alpha_{n}^{j} & \beta_{n}^{j} \\
\gamma_{n}^{j} & \delta_{n}^{j}
\end{array}\right)
$$

which describe simultaneously all 'positive' flows. In these terms equations (2.8)-(2.10) have the forms

$$
\begin{gathered}
b_{n+1}(\zeta)=\zeta b_{n}(\zeta)+\zeta r_{n}\left[d_{n+1}(\zeta)+d_{n}(\zeta)-i\right] \\
c_{n}(\zeta)=\zeta c_{n+1}(\zeta)+\zeta q_{n}\left[d_{n+1}(\zeta)+d_{n}(\zeta)-i\right] \\
d_{n+1}(\zeta)-d_{n}(\zeta)=q_{n} b_{n}(\zeta)-r_{n} c_{n+1}(\zeta)
\end{gathered}
$$

while equations (2.11) and (2.12) can be written as

$$
\partial(\zeta) q_{n}=q_{n} d_{n+1}(\zeta)+c_{n+1}(\zeta)
$$

and

$$
\partial(\zeta) r_{n}=-r_{n} d_{n}(\zeta)-b_{n}(\zeta)
$$

where

$$
\partial(\zeta)=\sum_{j=1}^{\infty} \zeta^{j} \partial_{j}
$$


It follows from (2.18)-(2.20) that the series $b_{n}(\zeta), c_{n}(\zeta)$ and $d_{n}(\zeta)$ are not independent. They are related by

$$
b_{n}(\zeta) c_{n}(\zeta)+\zeta d_{n}(\zeta)\left[d_{n}(\zeta)-i\right]=\text { constant }
$$

Using the invariance of the system (2.18)-(2.20) with respect to the transformations

$$
b_{n} \rightarrow(1+2 f) b_{n}, \quad c_{n} \rightarrow(1+2 f) c_{n}, \quad d_{n} \rightarrow(1+2 f) d_{n}-i f
$$

(with arbitrary $f$ ) we can eliminate the constant in the right-hand side of (2.24) and rewrite it as

$$
b_{n}(\zeta) c_{n}(\zeta)+\zeta d_{n}(\zeta)\left[d_{n}(\zeta)-i\right]=0 .
$$

This choice corresponds to the situation when we define $a_{n}^{0}=b_{n}^{0}=c_{n}^{0}=0, d_{n}^{0}=-i$ and do not introduce additional constants when we solve (2.10) for all $j$ 's, which leads to the, so to say, simplest form of the ALH equations (considered in [1]). Other choices lead to replacement of the ALH flows with their linear combinations.

Another consequence of (2.18)-(2.20) is that matrices $\mathcal{V}_{n}$ defined by

$$
\mathcal{V}_{n}(\lambda)=\left(\begin{array}{cc}
-d_{n}\left(\lambda^{2}\right) & \lambda^{-1} b_{n}\left(\lambda^{2}\right) \\
\lambda^{-1} c_{n}\left(\lambda^{2}\right) & d_{n}\left(\lambda^{2}\right)-i
\end{array}\right)
$$

satisfy the equation

$$
\mathcal{V}_{n+1}(\lambda) U_{n}(\lambda)-U_{n}(\lambda) \mathcal{V}_{n}(\lambda)=0,
$$

which is the stationary zero-curvature equation (2.4). Now one can express the matrices $V_{n}^{j}$ describing the ALH flows as

$$
V_{n}^{j}(\lambda)=\pi_{\lambda}^{+}\left[\lambda^{-2 j} \mathcal{V}_{n}(\lambda)\right]
$$

where $\pi_{\lambda}^{+}$is a projection operator defined by

$$
\pi_{\lambda}^{+}\left(\begin{array}{cc}
\sum_{j=-\infty}^{\infty} a_{j} \lambda^{2 j} & \sum_{j=-\infty}^{\infty} b_{j} \lambda^{2 j+1} \\
\sum_{j=-\infty}^{\infty} c_{j} \lambda^{2 j+1} & \sum_{j=-\infty}^{\infty} d_{j} \lambda^{2 j}
\end{array}\right)=\left(\begin{array}{cc}
\sum_{j=-\infty}^{-1} a_{j} \lambda^{2 j} & \sum_{j=-\infty}^{-1} b_{j} \lambda^{2 j+1} \\
\sum_{j=-\infty}^{-1} c_{j} \lambda^{2 j+1} & \sum_{j=-\infty}^{0} d_{j} \lambda^{2 j}
\end{array}\right) .
$$

Thus we have a usual situation for the case of 'integrable mathematics'. The space of the matrices involved in our hierarchy, $G_{\lambda}$, can be in a natural way decomposed in two subspaces $G_{\lambda}=\pi_{\lambda}^{+} G_{\lambda} \oplus \pi_{\lambda}^{-} G_{\lambda}$ where $\pi_{\lambda}^{-}$is given by

$$
\pi_{\lambda}^{-}\left(\begin{array}{cc}
\sum_{j=-\infty}^{\infty} a_{j} \lambda^{2 j} & \sum_{j=-\infty}^{\infty} b_{j} \lambda^{2 j+1} \\
\sum_{j=-\infty}^{\infty} c_{j} \lambda^{2 j+1} & \sum_{j=-\infty}^{\infty} d_{j} \lambda^{2 j}
\end{array}\right)=\left(\begin{array}{cc}
\sum_{j=0}^{\infty} a_{j} \lambda^{2 j} & \sum_{j=0}^{\infty} b_{j} \lambda^{2 j+1} \\
\sum_{j=0}^{\infty} c_{j} \lambda^{2 j+1} & \sum_{j=1}^{\infty} d_{j} \lambda^{2 j}
\end{array}\right),
$$

and all flows of the hierarchy can be constructed by projection onto one of them.

The matrix $\mathcal{V}_{n}(\lambda)$ plays a key role in the considerations below since it can be used to construct a (formal) solution of the linear system (2.1) and (2.5) (note that equation (2.28) is closely related to problem (2.1)). 


\section{Derivation of the functional equations}

The aim of this section is to obtain the functional equations which are equivalent to the ALH. As we mentioned in the introduction, in this paper we use an approach different from that of the work [1] and now we want to expose some differential properties of the series 2.17) which are 'hidden' in the recurrence relation (2.6).

Using (2.11), (2.12) together with (2.8), (2.9) one can obtain the following relations,

$$
\begin{aligned}
& \partial_{k} \beta_{n}^{j}-\partial_{k+1} \beta_{n}^{j-1}=\beta_{n}^{j} \delta_{n}^{k}+\beta_{n}^{j-1} \delta_{n}^{k+1}-2 \delta_{n}^{j-1} \beta_{n}^{k+1}, \\
& \partial_{k} \gamma_{n}^{j}-\partial_{k+1} \gamma_{n}^{j-1}=-\gamma_{n}^{j} \delta_{n}^{k}-\gamma_{n}^{j-1} \delta_{n}^{k+1}+2 \delta_{n}^{j-1} \gamma_{n}^{k+1}
\end{aligned}
$$

which, after replacing $j, k$ with $j+l, k-l$ and summing over $l$, become

$$
\begin{aligned}
& \partial_{k} \beta_{n}^{j}=i \beta_{n}^{j+k}-\beta_{n}^{j} \delta_{n}^{k}+2 \delta_{n}^{j} \beta_{n}^{k}+2 \sum_{l=1}^{j}\left(\beta_{n}^{l} \delta_{n}^{j+k-l}-\delta_{n}^{l} \beta_{n}^{j+k-p}\right), \\
& \partial_{k} \gamma_{n}^{j}=-i \gamma_{n}^{j+k}+\gamma_{n}^{j} \delta_{n}^{k}-2 \delta_{n}^{j} \gamma_{n}^{k}+2 \sum_{l=1}^{j}\left(\delta_{n}^{l} \gamma_{n}^{j+k-l}-\gamma_{n}^{l} \delta_{n}^{j+k-l}\right) .
\end{aligned}
$$

From these relations one can obtain expressions for $\partial(\zeta) b_{n}(\zeta)$ and $\partial(\zeta) c_{n}(\zeta)$. At first glance the latter is nonlocal because of the last terms in the right-hand sides of (3.3) and (3.4). However, this nonlocality can be quickly eliminated by means of the operator $\mathrm{d} / \mathrm{d} \zeta$. Indeed, using the identities

$$
\sum_{j, k=1}^{\infty} \zeta^{j+k} \sum_{l=1}^{j} u_{l} v_{j+k-l}=\sum_{l=1}^{\infty} \zeta^{l} u_{l} \sum_{j, k=1}^{\infty} \zeta^{j+k} v_{j+k}
$$

and

$$
\sum_{j, k=1}^{\infty} \zeta^{j+k} v_{j+k}=\sum_{m=2}^{\infty}(m-1) \zeta^{m} v_{m}=\left(\zeta \frac{\mathrm{d}}{\mathrm{d} \zeta}-1\right) \sum_{j=1}^{\infty} \zeta^{j} v_{j}
$$

one can derive from $(3.3)$ and $(3.4)$

$$
\begin{aligned}
& \partial(\zeta) b_{n}(\zeta)=b_{n}(\zeta)\left[d_{n}(\zeta)-i\right]+2 b_{n}(\zeta) \zeta \frac{\mathrm{d}}{\mathrm{d} \zeta} d_{n}(\zeta)+\left[i-2 d_{n}(\zeta)\right] \zeta \frac{\mathrm{d}}{\mathrm{d} \zeta} b_{n}(\zeta) \\
& \partial(\zeta) c_{n}(\zeta)=c_{n}(\zeta)\left[i-d_{n}(\zeta)\right]-2 c_{n}(\zeta) \zeta \frac{\mathrm{d}}{\mathrm{d} \zeta} d_{n}(\zeta)+\left[2 d_{n}(\zeta)-i\right] \zeta \frac{\mathrm{d}}{\mathrm{d} \zeta} c_{n}(\zeta)
\end{aligned}
$$

On the other hand it follows from (2.8)-(2.12) that

$$
\partial(\eta) d_{n}(\xi)=\frac{1}{\xi-\eta}\left\{b_{n}(\xi) c_{n}(\eta)-c_{n}(\xi) b_{n}(\eta)\right\}
$$

which leads to

$$
\partial(\zeta) d_{n}(\zeta)=c_{n}(\zeta) \frac{\mathrm{d}}{\mathrm{d} \zeta} b_{n}(\zeta)-b_{n}(\zeta) \frac{\mathrm{d}}{\mathrm{d} \zeta} c_{n}(\zeta)
$$

Note that expressions (3.7), (3.8) and (3.10) relate the evolutionary flows (I mean the differentiations, $\partial_{j}$, of the operator $\partial(\zeta)$ ) with the, so to say, 'auxiliary' (or 'spectral') one, $\mathrm{d} / \mathrm{d} \zeta$. X-Mozilla-Status: 0000 
Combining now (3.7), (3.8) and (3.10) one can obtain the following result:

$$
\begin{gathered}
\partial(\zeta) \frac{d_{n}(\zeta)}{b_{n}(\zeta)}=-i \zeta \frac{\mathrm{d}}{\mathrm{d} \zeta} \frac{d_{n}(\zeta)}{b_{n}(\zeta)}, \\
\partial(\zeta) \frac{d_{n}(\zeta)}{c_{n}(\zeta)}=i \zeta \frac{\mathrm{d}}{\mathrm{d} \zeta} \frac{d_{n}(\zeta)}{c_{n}(\zeta)}
\end{gathered}
$$

This means that the ratio $d_{n} / b_{n}$ (resp. $d_{n} / c_{n}$ ) depends on $z^{k}+i \zeta^{k} / k$ (resp. $\left.z^{k}-i \zeta^{k} / k\right)$. Noting also that

$$
\begin{gathered}
\lim _{\zeta \rightarrow 0} \frac{d_{n}(\zeta)}{b_{n}(\zeta)}=\frac{\delta_{n}^{1}}{\beta_{n}^{1}}=-q_{n}, \\
\lim _{\zeta \rightarrow 0} \frac{d_{n}(\zeta)}{c_{n}(\zeta)}=\frac{\delta_{n}^{1}}{\gamma_{n}^{1}}=-r_{n-1}
\end{gathered}
$$

one can present $d_{n} / b_{n}$ and $d_{n} / b_{n}$ as

$$
\begin{gathered}
\frac{d_{n}(z, \zeta)}{b_{n}(z, \zeta)}=-q_{n}(z+i[\zeta]), \\
\frac{d_{n}(z, \zeta)}{c_{n}(z, \zeta)}=-r_{n-1}(z-i[\zeta]),
\end{gathered}
$$

where the designation $z+\varepsilon[\zeta]$ stands for

$$
f(z+\varepsilon[\zeta])=f\left(z_{1}+\varepsilon \zeta, z_{2}+\varepsilon \zeta^{2} / 2, z_{3}+\varepsilon \zeta^{3} / 3, \ldots\right)
$$

and the dependence on the $\bar{z}_{j}$ is not indicated explicitly. Using (3.15)-(3.16) one can rewrite (2.18)-(2.20) as

$$
\begin{aligned}
& q_{n}(z+i[\zeta])-q_{n}(z)-\zeta \frac{c_{n+1}(z, \zeta)}{b_{n}(z, \zeta)}\left[1-q_{n}(z) r_{n}(z-i[\zeta])\right] r_{n-1}(z-i[\zeta])=0 \\
& r_{n}(z-i[\zeta])-r_{n}(z)-\zeta \frac{b_{n}(z, \zeta)}{c_{n+1}(z, \zeta)}\left[1-q_{n}(z+i[\zeta]) r_{n}(z)\right] q_{n+1}(z+i[\zeta])=0
\end{aligned}
$$

and

$$
b_{n}(z, \zeta)\left[q_{n}(z+i[\zeta])-q_{n}(z)\right]+c_{n+1}(z, \zeta)\left[r_{n}(z)-r_{n}(z-i[\zeta])\right]=0,
$$

from which it follows that

$$
\begin{aligned}
& q_{n}(z)-q_{n}(z-i[\zeta])=\zeta\left[1-q_{n}(z) r_{n}(z-i[\zeta])\right] q_{n+1}(z) \\
& r_{n}(z)-r_{n}(z+i[\zeta])=\zeta\left[1-q_{n}(z+i[\zeta]) r_{n}(z)\right] r_{n-1}(z)
\end{aligned}
$$

These equations (which were absent in explicit form in [1]) can be viewed as a functional representation of the 'positive' Ablowitz-Ladik subhierarchy. Expanding them in power series in $\zeta$ one can consequently obtain

$$
\begin{gathered}
\partial_{1} q_{n}=-i p_{n} q_{n+1}, \\
\partial_{1} r_{n}=i p_{n} r_{n-1}
\end{gathered}
$$


(the terms proportional to $\zeta^{1}$ ), then

$$
\begin{aligned}
\left(\partial_{2}-i \partial_{11}\right) q_{n} & =2 q_{n} q_{n+1} \partial_{1} r_{n}, \\
\left(\partial_{2}+i \partial_{11}\right) r_{n} & =2 r_{n-1} r_{n} \partial_{1} q_{n}
\end{aligned}
$$

(the terms proportional to $\zeta^{2}$ ), which using equations (3.23), (3.24) can be transformed to

$$
\begin{aligned}
& \partial_{2} q_{n}=i r_{n-1} p_{n} q_{n} q_{n+1}+i p_{n} r_{n} q_{n+1}^{2}-i p_{n} p_{n+1} q_{n+2}, \\
& \partial_{2} r_{n}=i r_{n-2} p_{n-1} p_{n}-i r_{n-1}^{2} p_{n} q_{n}-i r_{n-1} p_{n} r_{n} q_{n+1}
\end{aligned}
$$

and all other equations in the similar way.

The functional equations for the 'negative' subhierarchy can be written as

$$
\begin{aligned}
& q_{n}(\bar{z})-q_{n}\left(\bar{z}-i\left[\zeta^{-1}\right]\right)=\zeta^{-1}\left[1-q_{n}(\bar{z}) r_{n}\left(\bar{z}-i\left[\zeta^{-1}\right]\right)\right] q_{n-1}(\bar{z}), \\
& r_{n}(\bar{z})-r_{n}\left(\bar{z}+i\left[\zeta^{-1}\right]\right)=\zeta^{-1}\left[1-q_{n}\left(\bar{z}+i\left[\zeta^{-1}\right]\right) r_{n}(\bar{z})\right] r_{n+1}(\bar{z})
\end{aligned}
$$

with the dependence on the $z_{k}$ being omitted.

\section{Functional equations for tau-functions}

The functional equations obtained in the previous section, strictly speaking, complete the solution of the problem formulated in the title of this paper: equations (3.21), (3.22) and (3.29), (3.30) are sufficient to generate all equations of the ALH. However, we proceed further and present some results which seem to be interesting from various viewpoints.

It is widely known that intrinsic properties of integrable equations and hierarchies become more transparent when one uses Hirota's bilinear representation, i.e. when one operates not with the solutions themselves (the $q_{n} \mathrm{~s}$ and $r_{n} \mathrm{~s}$ in our case) but with the so-called tau-functions. The tau-functions of the ALH are defined by

$$
p_{n}=\frac{\tau_{n-1} \tau_{n+1}}{\tau_{n}^{2}}, \quad q_{n}=\frac{\sigma_{n}}{\tau_{n}}, \quad r_{n}=\frac{\rho_{n}}{\tau_{n}} .
$$

The definition of $p_{n}, p_{n}=1-q_{n} r_{n}$, rewritten in terms of tau functions, leads to the following relation between them:

$$
\tau_{n-1} \tau_{n+1}=\tau_{n}^{2}-\sigma_{n} \rho_{n} .
$$

Our aim now is to derive the functional equations for $\tau_{n}, \sigma_{n}$ and $\rho_{n}$ using equations obtained in the previous section. To this end we rewrite (3.21) and (3.22) as

$$
\begin{aligned}
& q_{n}^{+}-q_{n}=\zeta X_{n} q_{n+1}^{+}, \\
& r_{n}-r_{n}^{+}=\zeta X_{n} r_{n-1}
\end{aligned}
$$

with

$$
X_{n}=1-q_{n}^{+} r_{n}
$$

where the designation

$$
f_{n}^{+} \equiv f_{n}(z+i[\zeta], \bar{z})
$$


is used. It follows from (4.3) and (4.4) that the quantity $X_{n}$ satisfies the identities

$$
\begin{aligned}
X_{n}=1-r_{n}\left(q_{n}+\zeta X_{n} q_{n+1}^{+}\right) & =p_{n}-\zeta r_{n} q_{n+1}^{+} X_{n} \\
=1-q_{n}^{+}\left(r_{n}^{+}+\zeta X_{n} r_{n-1}\right) & =p_{n}^{+}-\zeta r_{n-1} q_{n}^{+} X_{n}
\end{aligned}
$$

which yields

$$
X_{n}=\frac{p_{n}}{1+\zeta r_{n} q_{n+1}^{+}}=\frac{p_{n}^{+}}{1+\zeta r_{n-1} q_{n}^{+}}
$$

and

$$
\frac{X_{n}}{X_{n+1}}=\frac{p_{n}}{p_{n+1}^{+}}=\frac{\tau_{n-1} \tau_{n+1}^{+}}{\tau_{n} \tau_{n}^{+}} \cdot \frac{\tau_{n+1} \tau_{n+1}^{+}}{\tau_{n} \tau_{n+2}^{+}} .
$$

From the last equation one can easily obtain that

$$
X_{n}=x \frac{\tau_{n-1} \tau_{n+1}^{+}}{\tau_{n} \tau_{n}^{+}}, \quad x=\text { constant }
$$

In what follows we restrict our attention to the case

$$
x=1
$$

which corresponds to the so-called 'finite-density' boundary conditions

$$
\lim _{n \rightarrow \pm \infty} p_{n}=\text { constant }
$$

(which also includes the vanishing boundary conditions considered in [1]). In some other important cases, first of all the case when $x=x(\zeta)$ depends on $\zeta$ but does not depend on the $z_{j}$ s (such constant $x$ appears in the quasi-periodic situation), one can construct the substitution $\left(\tau_{n}, \sigma_{n}, \rho_{n}\right) \rightarrow \exp \left\{\alpha n^{2}+n f_{1}(z)+f_{0}(z)\right\}\left(\tau_{n}, \sigma_{n}, \rho_{n}\right)$, where $f_{1,0}(z)$ are some linear function of the $z_{j} \mathrm{~s}$, which eliminates the constant $x(\zeta)$ and hence provides reduction to the case of this paper. X-Mozilla-Status: 0000

Substituting (4.11) in (4.5) and then in (4.3), (4.4) one can obtain the equations

$$
\begin{aligned}
& \tau_{n}(z) \tau_{n}(z+i[\zeta])-\rho_{n}(z) \sigma_{n}(z+i[\zeta])=\tau_{n-1}(z) \tau_{n+1}(z+i[\zeta]), \\
& \tau_{n}(z) \sigma_{n}(z+i[\zeta])-\sigma_{n}(z) \tau_{n}(z+i[\zeta])=\zeta \tau_{n-1}(z) \sigma_{n+1}(z+i[\zeta]), \\
& \rho_{n}(z) \tau_{n}(z+i[\zeta])-\tau_{n}(z) \rho_{n}(z+i[\zeta])=\zeta \rho_{n-1}(z) \tau_{n+1}(z+i[\zeta]),
\end{aligned}
$$

where dependence on the $\bar{z}_{j} \mathrm{~s}$ is omitted. This system was the main result of the paper [1]. In what follows we use some other form of (4.14)-(4.16), which can be obtained from the latter by means of (4.2):

$$
\begin{aligned}
& \tau_{n+1}(z) \tau_{n}(z+i[\zeta])-\tau_{n}(z) \tau_{n+1}(z+i[\zeta])=\zeta \rho_{n}(z) \sigma_{n+1}(z+i[\zeta]), \\
& \tau_{n+1}(z) \sigma_{n}(z+i[\zeta])-\sigma_{n}(z) \tau_{n+1}(z+i[\zeta])=\zeta \tau_{n}(z) \sigma_{n+1}(z+i[\zeta]), \\
& \rho_{n+1}(z) \tau_{n}(z+i[\zeta])-\tau_{n}(z) \rho_{n+1}(z+i[\zeta])=\zeta \rho_{n}(z) \tau_{n+1}(z+i[\zeta]) .
\end{aligned}
$$

Before proceeding further we present, without derivation, the equations which are equivalent to the 'negative' subhierarchy. The systems analogous to (4.14) $-(4.16)$ and 
(4.17) $-(4.19)$ in the 'negative' case can be written as

$$
\begin{gathered}
\tau_{n}(\bar{z}) \tau_{n}\left(\bar{z}+i\left[\zeta^{-1}\right]\right)-\rho_{n}(\bar{z}) \sigma_{n}\left(\bar{z}+i\left[\zeta^{-1}\right]\right) \\
=\tau_{n+1}(\bar{z}) \tau_{n-1}\left(\bar{z}+i\left[\zeta^{-1}\right]\right), \\
\tau_{n}(\bar{z}) \sigma_{n}\left(\bar{z}+i\left[\zeta^{-1}\right]\right)-\sigma_{n}(\bar{z}) \tau_{n}\left(\bar{z}+i\left[\zeta^{-1}\right]\right) \\
=\zeta^{-1} \tau_{n+1}(\bar{z}) \sigma_{n-1}\left(\bar{z}+i\left[\zeta^{-1}\right]\right), \\
\rho_{n}(\bar{z}) \tau_{n}\left(\bar{z}+i\left[\zeta^{-1}\right]\right)-\tau_{n}(\bar{z}) \rho_{n}\left(\bar{z}+i\left[\zeta^{-1}\right]\right) \\
=\zeta^{-1} \rho_{n+1}(\bar{z}) \tau_{n-1}\left(\bar{z}+i\left[\zeta^{-1}\right]\right)
\end{gathered}
$$

and

$$
\begin{gathered}
\tau_{n-1}(\bar{z}) \tau_{n}\left(\bar{z}+i\left[\zeta^{-1}\right]\right)-\tau_{n}(\bar{z}) \tau_{n-1}\left(\bar{z}+i\left[\zeta^{-1}\right]\right) \\
=\zeta^{-1} \rho_{n}(\bar{z}) \sigma_{n-1}\left(\bar{z}+i\left[\zeta^{-1}\right]\right) \\
\tau_{n-1}(\bar{z}) \sigma_{n}\left(\bar{z}+i\left[\zeta^{-1}\right]\right)-\sigma_{n}(\bar{z}) \tau_{n-1}\left(\bar{z}+i\left[\zeta^{-1}\right]\right) \\
=\zeta^{-1} \tau_{n}(\bar{z}) \sigma_{n-1}\left(\bar{z}+i\left[\zeta^{-1}\right]\right) \\
\rho_{n-1}(\bar{z}) \tau_{n}\left(\bar{z}+i\left[\zeta^{-1}\right]\right)-\tau_{n}(\bar{z}) \rho_{n-1}\left(\bar{z}+i\left[\zeta^{-1}\right]\right) \\
=\zeta^{-1} \rho_{n}(\bar{z}) \tau_{n-1}\left(\bar{z}+i\left[\zeta^{-1}\right]\right) .
\end{gathered}
$$

Returning to the 'positive' subhierarchy, we note that equations (4.14)-(4.16) contain much information about the ALH and we now derive some of their consequences. Firstly we continue discussion of the zero-curvature representation of the ALH started in Section 2. The relation (2.28) indicates that the matrix $\mathcal{V}_{n}$ can be presented in the form $\mathcal{V}_{n}=$ $\Phi_{n} C \Phi_{n}^{-1}$, where $C$ is a constant (with respect to $n$ ) matrix and $\Phi_{n}$ is a solution of the scattering problem (2.1). Thus one can hope to obtain from (2.27) some, at least formal, solution of the scattering problem, which can be then modified to become a solution of the evolutionary problem (2.5) as well, i.e. to obtain the Baker-Akhiezer function of our problem. One can hardly derive a closed local expression for $\Phi_{n}$ in terms of the quantities $b_{n}, c_{n}$ and $d_{n}$, but this can be done in terms of the tau-functions.

Consider the matrix

$$
F_{n}(z, \bar{z}, \lambda)=\frac{1}{\tau_{n-1}(z, \bar{z})}\left(\begin{array}{cc}
\tau_{n}\left(z+i\left[\lambda^{2}\right], \bar{z}\right) & \lambda \rho_{n-1}\left(z-i\left[\lambda^{2}\right], \bar{z}\right) \\
-\lambda \sigma_{n}\left(z+i\left[\lambda^{2}\right], \bar{z}\right) & \tau_{n-1}\left(z-i\left[\lambda^{2}\right], \bar{z}\right)
\end{array}\right) .
$$

Using (4.14)-(4.19) one can straightforwardly verify that this matrix satisfies the equation

$$
U_{n}(\lambda) F_{n}(\lambda)=F_{n+1}(\lambda)\left(\begin{array}{cc}
\lambda & 0 \\
0 & \lambda^{-1}
\end{array}\right)
$$

which means that

$$
\Phi_{n}(z, \bar{z}, \lambda)=F_{n}(z, \bar{z}, \lambda)\left(\begin{array}{cc}
\lambda^{n} & 0 \\
0 & \lambda^{-n}
\end{array}\right)
$$

is a (formal) solution of (2.1).

Analogously from the 'negative' subhierarchy one can derive another solution of (4.27), $\bar{F}_{n}$,

$$
\bar{F}_{n}(z, \bar{z}, \lambda)=\frac{1}{\tau_{n-1}(z, \bar{z})}\left(\begin{array}{cc}
\tau_{n-1}\left(z, \bar{z}+i\left[\lambda^{-2}\right]\right) & -\lambda^{-1} \rho_{n}\left(z, \bar{z}-i\left[\lambda^{-2}\right]\right) \\
\lambda^{-1} \sigma_{n-1}\left(z, \bar{z}+i\left[\lambda^{-2}\right]\right) & \tau_{n}\left(z, \bar{z}-i\left[\lambda^{-2}\right]\right)
\end{array}\right)
$$


and, hence, another solution of the scattering problem:

$$
\bar{\Phi}_{n}(z, \bar{z}, \lambda)=\bar{F}_{n}(z, \bar{z}, \lambda)\left(\begin{array}{cc}
\lambda^{n} & 0 \\
0 & \lambda^{-n}
\end{array}\right)
$$

\section{Discrete-time Ablowitz-Ladik equations}

In Section 3 we have derived the functional equations (3.21), (3.22) and (3.29), (3.30) which we now rewrite as

$$
\begin{aligned}
& \widehat{q}_{n}-q_{n}=\xi\left[1-\widehat{q}_{n} r_{n}\right] \widehat{q}_{n+1}, \\
& r_{n}-\widehat{r}_{n}=\xi\left[1-\widehat{q}_{n} r_{n}\right] r_{n-1},
\end{aligned}
$$

where $\widehat{q}_{n}=q_{n}(z+i[\xi], \bar{z})$ etc and

$$
\begin{aligned}
& q_{n}-\widetilde{q}_{n}=\xi^{-1}\left[1-q_{n} \widetilde{r}_{n}\right] q_{n-1}, \\
& \widetilde{r}_{n}-r_{n}=\xi^{-1}\left[1-q_{n} \widetilde{r}_{n}\right] \widetilde{r}_{n+1}
\end{aligned}
$$

with $\widetilde{q}_{n}=q_{n}\left(z, \bar{z}-i\left[\xi^{-1}\right]\right)$ etc, and demonstrated that these equations are equivalent to the ALH. It should be noted that equations (5.1)-(5.4) have already been discussed in the literature in some other context. We mean the so-called discrete-time Ablowitz-Ladik equations, which arise as compatibility conditions for the discrete-discrete linear system

$$
\Psi_{n+1}=U_{n} \Psi_{n}
$$

and

$$
\widehat{\Psi}_{n}=M_{n} \Psi_{n}
$$

where (5.5) is the scattering problem of the ALH, and which are the integrable discretizations of the differential-difference Ablowitz-Ladik equations.

Before proceeding further, we note that equations (5.5) and (5.6) are essentially discrete equations. Strictly speaking they should be written as

$$
\Psi_{n+1, m}=U_{n, m} \Psi_{n, m} \quad \text { and } \quad \Psi_{n, m+1}=M_{n, m} \Psi_{n, m}
$$

and the resulting equations will take the form

$$
\begin{aligned}
& q_{n, m}-q_{n, m+1}=Q\left(q_{n, m}, r_{n, m}, q_{n \pm 1, m \pm 1}, r_{n \pm 1, m \pm 1}, \ldots\right) \\
& r_{n, m}-r_{n, m+1}=R\left(q_{n, m}, r_{n, m}, q_{n \pm 1, m \pm 1}, r_{n \pm 1, m \pm 1}, \ldots\right) .
\end{aligned}
$$

Depending upon the choice of the $M$-matrix one can derive equations which cannot be reduced by some limiting procedure to the evolutionary equations of the ALH (for example, the system (5.5) and (5.6) is the starting point for obtaining Bäcklund transformations). However, among equations (5.8) and (5.9) there is a big class of equations having the continuous limit, and we discuss here these equations, which were studied in [7, 4]. In the paper [1] by Suris it has been shown that the nonlocal scheme of Ablowitz and Ladik [7] can be factorized into the product of the local ones which are exactly (up to redefinition of variables) equations (5.1), (5.2) and (5.3), (5.4). Thus the results of the previous sections 
lead to a, so to say, explicit relation between the ALH and the equations of [1] in terms of the solutions: each solution of, for example, the 'positive' subhierarchy provides a solution of the discrete-time equations (5.1) and (5.2) after identifying $\widehat{q}_{n}$ and $\widehat{r}_{n}$ with $q_{n}(z+i[\xi], \bar{z})$ and $r_{n}(z+i[\xi], \bar{z})$. In other words the discrete flow is equivalent to (or at least can be modeled as) a properly chosen combination of the infinite number of continuous ones: the shift $\Psi_{n} \rightarrow \widehat{\Psi}_{n}$ can be obtained as simultaneous shifts in all $z_{k^{-}}$directions, $z_{k} \rightarrow z_{k}+i \xi^{k} / k$, $(k=1,2, \ldots)$.

This result is not new. It has been already obtained by Suris in [⿴囗十 in the framework of the Hamiltonian approach. One can find in [4] an explicit expression for the Hamiltonian of the discrete-time flow, from which it follows that it can be presented as a linear combination of the Hamiltonians of the differential Ablowitz-Ladik equations (Proposition 12 of [4]). From this standpoint the above consideration can be viewed as an alternative derivation of this result, which does not exploit Hamiltonian structures, uses only local properties of the ALH and, hence, is not so sensitive to the boundary conditions.

The main subject of this paper is the ALH, not equations (5.8) and (5.9) themselves. For us the representation (5.1)-(5.4) is a way to obtain some results for the AblowitzLadik equations by studying the discrete system which is, in some sense, a more simple object than the original system of differential equations. In [9] it was shown how this approach can be used to derive, without much effort, the finite-genus quasiperiodical solutions, the problem which is rather difficult technically if one solves it in the language of differential equations (see [3]). Firstly one solves the discrete equations (5.1)-(5.4) using Fay's identities for the $\theta$-functions and then adjusts some constants in such a way that the discrete shifts become Miwa's shifts $z_{k} \rightarrow z_{k}+i \xi^{k} / k, \bar{z}_{k} \rightarrow \bar{z}_{k}-i \xi^{-k} / k$.

In what follows we use the discrete time representation (5.5) and (5.6) to obtain the 'superposition' formulae for the tau-functions of the ALH. Thus we want to finish this section by writing down the $M$-matrices which lead to (5.1)-(5.4).

The matrix $M_{n}$ describing shifts in the 'positive' discrete direction, $(z \rightarrow z+i[\xi])$ can be written as

$$
M_{n}(z, \lambda ; \xi)=\frac{1}{\tau_{n} \widehat{\tau}_{n-1}}\left(\begin{array}{cc}
\tau_{n} \widehat{\tau}_{n-1}-\lambda^{-2} \xi \tau_{n-1} \widehat{\tau}_{n} & \lambda^{-1} \xi \rho_{n-1} \widehat{\tau}_{n} \\
\lambda^{-1} \xi \tau_{n-1} \widehat{\sigma}_{n} & \tau_{n-1} \widehat{\tau}_{n}
\end{array}\right) .
$$

Using the functional equations (4.14)-(4.19) for the quantities $\widehat{\tau}_{n}=\tau_{n}(z+i[\xi])$ etc, one can verify the relation

$$
M_{n+1}(\lambda ; \xi) U_{n}(\lambda)=\widehat{U}_{n}(\lambda) M_{n}(\lambda ; \xi)
$$

where

$$
\widehat{U}_{n}(\lambda)=\left(\begin{array}{cc}
\lambda & \widehat{r}_{n} \\
\widehat{q}_{n} & \lambda^{-1}
\end{array}\right)
$$

Analogously the 'negative' discrete-time shift matrices, $\bar{M}_{n}$, can be presented as

$$
\bar{M}_{n}(\bar{z}, \lambda ; \xi)=\frac{1}{\tau_{n} \widetilde{\tau}_{n-1}}\left(\begin{array}{cc}
\tau_{n-1} \widetilde{\tau}_{n} & \lambda \xi^{-1} \tau_{n-1} \widetilde{\rho}_{n} \\
\lambda \xi^{-1} \sigma_{n-1} \widetilde{\tau}_{n} & \tau_{n} \widetilde{\tau}_{n-1}-\lambda^{2} \xi^{-1} \tau_{n-1} \widetilde{\tau}_{n}
\end{array}\right)
$$

with $\widetilde{\tau}_{n}$ standing for $\tau_{n}\left(\bar{z}-i\left[\xi^{-1}\right]\right)$ etc. These matrices after substitution into $\bar{M}_{n+1} U_{n}=$ $\widetilde{U}_{n} \bar{M}_{n}$ lead to equations (5.3)-(5.4). 


\section{$6 \quad$ Fay's identities for the tau-functions}

The aim of this section is to derive some results for the ALH by means of, so to say, discrete-time approach. We do not use the evolutionary part of the ZCR, (2.2), and explicit form of the ALH flows. All we need now is the fact that their combined action can be presented as the map discussed in the previous section, and can be described by the $M$-matrices (5.10) or (5.13). The question, which we address now, is the superposition of the maps $q_{n} \rightarrow \hat{q}_{n}$ (or, in other words, superposition of Miwa's shifts) which enables us to obtain the so-called Fay's identities for the ALH.

The discrete-time zero-curvature condition (5.11), when considered in the framework of the functional equations for the ALH, is

$$
M_{n+1}(z, \lambda ; \xi) U_{n}(z, \lambda)=U_{n}(z+i[\xi], \lambda) M_{n}(z, \lambda ; \xi)
$$

(the dependence on $\bar{z}=\left(\bar{z}_{1}, \bar{z}_{2}, \ldots\right)$ is omitted). Consider now the matrices (4.28) which solve

$$
\Phi_{n+1}=U_{n} \Phi_{n}
$$

Acting on this equation by $M_{n+1}$ one can obtain

$$
M_{n+1} \Phi_{n+1}=\widehat{U}_{n} M_{n} \Phi_{n}, \quad \widehat{U}_{n} \equiv U_{n}(z+i[\xi], \lambda) .
$$

Thus the product $M_{n} \Phi_{n}$ differs from $\widehat{\Phi}_{n}$ only by constant matrix $C$ or, in terms of $F_{n}$ 's,

$$
M_{n} F_{n}=\widehat{F}_{n} \Lambda^{n} C \Lambda^{-n}, \quad \Lambda=\left(\begin{array}{cc}
\lambda & 0 \\
0 & \lambda^{-1}
\end{array}\right) .
$$

By direct calculation one can verify that the nondiagonal elements of the matrix $\widehat{F}_{n}^{-1} M_{n} F_{n}$ are zero,

$$
\begin{aligned}
& {\left[F_{n}^{-1}(z+i[\xi], \lambda) M_{n}(z, \lambda ; \xi) F_{n}(z, \lambda)\right]^{(12)}=0} \\
& {\left[F_{n}^{-1}(z+i[\xi], \lambda) M_{n}(z, \lambda ; \xi) F_{n}(z, \lambda)\right]^{(21)}=0}
\end{aligned}
$$

i.e. $C$ is a diagonal matrix, which hence commutes with $\Lambda$,

$$
M_{n}(z, \lambda ; \xi) F_{n}(z, \lambda)=F_{n}(z+i[\xi], \lambda)\left(\begin{array}{cc}
C_{1}(\zeta ; \xi) & 0 \\
0 & C_{2}(\zeta ; \xi)
\end{array}\right), \quad \zeta=\lambda^{2} .
$$

The upper-left element of this matrix relation can be written as

$$
\begin{gathered}
\kappa(\zeta, \xi) \tau_{n-1}(z) \tau_{n}(z+i[\zeta]+i[\xi]) \\
=\zeta \tau_{n}(z+i[\zeta]) \tau_{n-1}(z+i[\xi])-\xi \tau_{n}(z+i[\xi]) \tau_{n-1}(z+i[\zeta]),
\end{gathered}
$$

where

$$
\kappa(\zeta, \xi)=-\kappa(\xi, \zeta)=\zeta C_{1}(\zeta ; \xi)
$$

It can be shown that analogous identities can be derived for all tau-functions,

$$
\begin{gathered}
\kappa(\zeta, \xi) \omega_{n-1}(z) \omega_{n}(z+i[\zeta]+i[\xi]) \\
=\zeta \omega_{n}(z+i[\zeta]) \omega_{n-1}(z+i[\xi])-\xi \omega_{n}(z+i[\xi]) \omega_{n-1}(z+i[\zeta])
\end{gathered}
$$


where

$$
\omega_{n}=\sigma_{n}, \quad \rho_{n} \quad \text { or } \quad \tau_{n}
$$

These equations are the simplest 'determinant' identities for the 'positive' ALH. They can be generalized to obtain determinantal representation of superposition of arbitrary number of Miwa's shifts:

$$
\begin{gathered}
K\left(\zeta_{1}, \ldots, \zeta_{M}\right) \omega_{n}(z) \cdots \omega_{n+M-2}(z) \omega_{n+M-1}\left(z+i\left[\zeta_{1}\right]+\cdots+i\left[\zeta_{M}\right]\right) \\
=\operatorname{det}\left|\zeta_{r}^{s-1} \omega_{n+s-1}\left(z+i\left[\zeta_{r}\right]\right)\right|_{r, s=1, \ldots, M}
\end{gathered}
$$

where

$$
K\left(\zeta_{1}, \ldots, \zeta_{M}\right)=\prod_{1 \leq j<k \leq M} \kappa\left(\zeta_{k}, \zeta_{j}\right)
$$

Expanding the determinants (say, over the first few columns) one can obtain a large number of multilinear relations the simplest of which (one-column) are

$$
\begin{gathered}
\omega_{n-M+1}(z) \omega_{n}\left(z+i\left[\zeta_{1}\right]+\cdots+i\left[\zeta_{M}\right]\right) \\
=\sum_{j=1}^{M} K_{j} \omega_{n-M+1}\left(z+i\left[\zeta_{j}\right]\right) \omega_{n}\left(z+i\left[\zeta_{1}\right]+\cdots+\widehat{i\left[\zeta_{j}\right]}+\cdots+i\left[\zeta_{M}\right]\right) .
\end{gathered}
$$

The symbol ${ }^{\wedge}$ here indicates that the corresponding quantity should be omitted and

$$
K_{j}=\prod_{k \neq j}^{\prime} \frac{\zeta_{k}}{\kappa\left(\zeta_{k}, \zeta_{j}\right)} .
$$

Clearly analogous formulae can be derived for the 'negative' shifts as well.

\section{Integrable discretizations of higher flows and 'reduced' Miwa's shifts}

After we have derived the superposition formulae for Miwa's shifts, we can address the following question (which has been raised by the referee of this paper). The discretetime equation discussed in the Section 5 has been considered by Suris as the integrable discretization of the simplest Ablowitz-Ladik flows. At the same time it interpolates all continuous ALH flows. However it is possible to construct the integrable discretizations of the higher Ablowitz-Ladik flows as has been done, e.g., in the paper [8]. So the question is how these higher discrete-time equations can be related to the original ALH and to its Miwa's shift representation?

To get some insight into this problem consider the twice shifted tau-functions,

$$
\tilde{\omega}_{n}=\omega_{n}(z+i[\xi]+i[\eta]), \quad \omega_{n}=\sigma_{n}, \quad \rho_{n} \quad \text { or } \quad \tau_{n}
$$

One can derive from formulae $(6.10)$ the following identity

$$
\xi \eta\left(\rho_{n} \tilde{\varphi}_{n+1}-\tau_{n-1} \tilde{\sigma}_{n+2}\right)+(\xi+\eta) \tau_{n} \tilde{\sigma}_{n+1}+\sigma_{n} \tilde{\tau}_{n+1}-\tau_{n+1} \tilde{\sigma}_{n}=0
$$


where $\varphi_{n}$ is defined by

$$
\tau_{n} \varphi_{n}=\sigma_{n}^{2}-\sigma_{n-1} \sigma_{n+1}
$$

(in other words $\varphi_{n}$ is the next tau-function in the chain $\cdots \rightarrow \rho_{n} \rightarrow \tau_{n} \rightarrow \sigma_{n} \rightarrow \varphi_{n} \rightarrow \cdots$, compare with (4.2)). After the imposition of the restriction

$$
\eta=-\xi
$$

the relation $(7.2)$ leads to

$$
\frac{\tilde{q}_{n}-q_{n}}{\xi^{2}}=P_{n}\left(\tilde{p}_{n+1} \tilde{q}_{n+2}-r_{n} \tilde{q}_{n+1}^{2}-r_{n-1} q_{n} \tilde{q}_{n+1}-\xi^{2} r_{n-1} P_{n} \tilde{p}_{n+1} \tilde{q}_{n+1} \tilde{q}_{n+2}\right)
$$

and

$$
\frac{r_{n}-\tilde{r}_{n}}{\xi^{2}}=P_{n}\left(r_{n-2} p_{n-1}-r_{n-1}^{2} \tilde{q}_{n}-r_{n-1} \tilde{r}_{n} \tilde{q}_{n+1}-\xi^{2} r_{n-2} r_{n-1} p_{n-1} P_{n} \tilde{q}_{n+1}\right),
$$

where

$$
P_{n}=P_{n}(\xi)=\frac{1-r_{n} \tilde{q}_{n}}{1+\xi^{2} r_{n-1} \tilde{q}_{n+1}} .
$$

If one considers $q \rightarrow \tilde{q}$ as the discrete-time shift, then equations (7.5) and (7.6) form a closed discrete-time system. On the other hand

$$
\tilde{q}, \tilde{r}=q, r\left(z_{1}, z_{2}+i \xi^{2}, \ldots\right)
$$

and in the $\xi \rightarrow 0$ limit the left-hand sides of (7.5) and (7.6) become $i \partial q / \partial z_{2}$ and $i \partial r / \partial z_{2}$, while the right-hand sides become the expressions for the second positive ALH flow, see (3.27) and (3.28). Thus the system (7.5) and (7.6) can be viewed as an integrable discretization of the second ALH flow and, indeed, this system is a condensed form of the corresponding equations of the work [8].

In other words the second discrete-time flow can be constructed using the product of two Miwa's shifts $T_{z}(\xi) T_{z}(-\xi)$, where

$$
T_{z}(\xi): \quad z_{k} \rightarrow z_{k}+i \xi^{k} / k .
$$

Looking once more on the structure of the double shifted functions

$$
\tilde{q}, \tilde{r}=q, r\left(z_{1}, z_{2}+i \xi^{2}, z_{3}, z_{4}+i \xi^{2} / 2, \ldots\right)
$$

we easily note that the odd $z_{k}$ 's remain unchanged, while the even variables gain shifts proportional to powers of $\xi^{2}$,

$$
T_{z}^{(2)}(\xi)=T_{z}(\xi) T_{z}(-\xi): \quad\left\{\begin{array}{l}
z_{2 k-1} \rightarrow z_{2 k-1} \\
z_{2 k} \rightarrow z_{2 k}+i \xi^{2 k} / k
\end{array}\right.
$$

So, if one forgets for a while about the odd $z_{k}$ and introduces "even" variables, $y_{k}$, by $y_{k}=z_{2 k}$, then the action of the binary Miwa's shift $T_{z}^{(2)}(\xi)$ can be presented as the action of the usual Miwa's shift with squared parameter,

$$
T_{y}\left(\xi^{2}\right): \quad y_{k} \rightarrow y_{k}+i\left(\xi^{2}\right)^{k} / k .
$$


Thus we can consider $T_{z}(\xi) T_{z}(-\xi)$ as some kind of "reduced" Miwa's shift, which generates the second discrete-time flow.

This construction can be extended. Introducing the $M$ th-order product of $T_{z}$,

$$
T_{z}^{(M)}(\xi)=\prod_{\ell=0}^{M-1} T_{z}\left(\epsilon^{\ell} \xi\right), \quad \epsilon^{M}=1,
$$

one can easily conclude that this operator affects only each $M$ th variable,

$$
T_{z}^{(M)}(\xi): \quad\left\{\begin{array}{l}
z_{k} \rightarrow z_{k} \quad \text { for } \quad k \neq M l \\
z_{M l} \rightarrow z_{M l}+i \xi^{M l} / l
\end{array}\right.
$$

So, if we construct equations similar to 7.5$)$ and $(7.6)$,

$$
\frac{\tilde{q}_{n}-q_{n}}{\xi^{M}}=\cdots \quad \text { and } \quad \frac{r_{n}-\tilde{r}_{n}}{\xi^{M}}=\cdots
$$

where $\tilde{q}_{n}(z)=T_{z}^{(M)}(\xi) q_{n}(z), \tilde{r}_{n}(z)=T_{z}^{(M)}(\xi) r_{n}(z)$, then in the $\xi \rightarrow 0$ limit they become the $M$ th Ablowitz-Ladik equation. Thus the $M$ th discrete-time flow is produced by $T_{z}^{(M)}(\xi)$ which after introducing variables

$$
y_{m}^{(M)}=z_{M m}
$$

can be rewritten as the usual Miwa's shift with the $M$ th power of the parameter:

$$
T_{y^{(M)}}\left(\xi^{M}\right): \quad y_{k} \rightarrow y_{k}+i\left(\xi^{M}\right)^{k} / k
$$

However, to construct equations of the type (7.15) starting from the superposition formulae $(6.10)$ is a rather cumbersome procedure and, if one wants to derive the $M$ th discrete-time Ablowitz-Ladik equation, it is easier to use the direct, zero-curvature, approach of the works [0, 4,8$]$.

\section{Conservation laws}

As we mentioned in the Introduction, in this section we discuss an example of the practical applications of the functional representation of the ALH and consider once more the question of describing the infinite series of the conservation laws of this integrable model.

The conserved quantities of the ALH have been known since the work of Ablowitz and Ladik [10] and one can find there (or in a textbook; see, e.g., [11]) how to derive them as well as their generating function. However, this generating function is usually given in terms of the solutions of the auxiliary problem (2.1) (i.e. the Jost functions) while one usually wants to write the conservation laws in terms of the solutions of the equations of the hierarchy themselves. Now we know how, using Miwa's shifts, to express the former in terms of the latter. So we can describe the conserved quantities (and corresponding divergence-like formulae) using directly the tau-functions of the ALH. 
The first constants of motion are given by

$$
\begin{gathered}
I_{1}=\sum_{n} r_{n-1} q_{n}, \\
I_{2}=\sum_{n} r_{n-1} p_{n} q_{n+1}-\frac{1}{2} r_{n-1}^{2} q_{n}^{2} .
\end{gathered}
$$

One can easily observe that the summands in these formulae are nothing but the quantities $\delta_{n}^{j}$ from (2.6), videlicet

$$
\begin{gathered}
\delta_{n}^{1}=i r_{n-1} q_{n}, \\
\delta_{n}^{2}=i r_{n-2} p_{n-1} q_{n}+i r_{n-1} p_{n} q_{n+1}-i r_{n-1}^{2} q_{n}^{2} .
\end{gathered}
$$

Indeed, as follows from (2.11), (2.12) and (2.8)-(2.10), one can present the $\delta_{n}^{j}$ as

$$
\delta_{n}^{j}=\partial_{j} \ln \frac{\tau_{n}}{\tau_{n-1}} .
$$

Hence

$$
\partial_{k} \delta_{n}^{j}=\partial_{j k} \ln \tau_{n}-\partial_{j k} \ln \tau_{n-1} .
$$

Obviously the right-hand side of this identity vanishes after summation over $n$ in the case of an infinite chain under the zero or finite-density boundary conditions (or in a periodic case). Thus the series $d_{n}(\zeta)=\sum_{j=1}^{\infty} \zeta^{j} \delta_{n}^{j}$ (see 2.17) ) can be viewed as a generating function for the constants of motion. To complete the description of the conservation laws,

$$
\partial_{k} \delta_{n}^{j}=f_{n}^{j k}-f_{n-1}^{j k}
$$

we have to obtain an expression (and a generating function) for the quantities $f_{n}^{j k}=$ $\partial_{j k} \ln \tau_{n}$. To this end consider equation (3.9). After some algebraic manipulations with (2.18)-(2.20) one can rewrite (3.9) in the form which we need:

$$
\partial(\eta) d_{n}(\xi)=f_{n}(\xi, \eta)-f_{n-1}(\xi, \eta)
$$

where

$$
\begin{array}{r}
f_{n}(\xi, \eta)=\frac{\xi \eta}{(\xi-\eta)^{2}}\left[2 d_{n}(\xi) d_{n}(\eta)-i d_{n}(\xi)-i d_{n}(\eta)\right. \\
\left.\quad+\xi^{-1} b_{n}(\xi) c_{n}(\eta)+\eta^{-1} b_{n}(\eta) c_{n}(\xi)\right]
\end{array}
$$

This expression, after expansion in $\xi$ and $\eta$, gives (8.7) with

$$
f_{n}^{j k}=-\sum_{p=1}^{j} \sum_{q=1}^{k}\left[\delta_{n+1}^{p+q-1} \delta_{n+1}^{j+k+1-p-q}+\beta_{n+1}^{p+q} \gamma_{n+1}^{j+k+1-p-q}\right] .
$$

These formulae solve our problem. However, one may feel some dissatisfaction with their form. The results obtained can be written in a more elegant way if one expresses them in 
the terms of the tau-functions with shifted arguments. To do this one needs the following formulae for $b_{n}(\zeta), c_{n}(\zeta)$ and $d_{n}(\zeta)$ which can be derived from (2.20) and (3.15), (3.16):

$$
\begin{aligned}
& b_{n}(\zeta)=-d_{*}(\zeta) \frac{\rho_{n-1}(z-i[\zeta]) \tau_{n}(z+i[\zeta])}{\tau_{n-1}(z) \tau_{n}(z)} \\
& c_{n}(\zeta)=-d_{*}(\zeta) \frac{\tau_{n-1}(z-i[\zeta]) \sigma_{n}(z+i[\zeta])}{\tau_{n-1}(z) \tau_{n}(z)} \\
& d_{n}(\zeta)=d_{*}(\zeta) \frac{\rho_{n-1}(z-i[\zeta]) \sigma_{n}(z+i[\zeta])}{\tau_{n-1}(z) \tau_{n}(z)}
\end{aligned}
$$

Here $d_{*}(\zeta)$ is some constant (with respect to the index $n$ ) which appears when one solves (2.20) and which depends on the boundary conditions for the $q_{n}$ and $r_{n}$. Using these relations together with the functional equations (4.14)-(4.19) and the 'determinant' identities (6.10) one can rewrite the conservation laws as follows:

$$
\partial(\eta) \mathcal{H}_{n}(z, \bar{z} ; \xi)=\mathcal{F}_{n}(z, \bar{z} ; \xi, \eta)-\mathcal{F}_{n-1}(z, \bar{z} ; \xi, \eta)
$$

where

$$
\mathcal{H}_{n}(z, \bar{z} ; \xi)=\frac{\rho_{n-1}(z-i[\xi], \bar{z}) \sigma_{n}(z+i[\xi], \bar{z})}{\tau_{n-1}(z, \bar{z}) \tau_{n}(z, \bar{z})}
$$

and

$$
\mathcal{F}_{n}(z, \bar{z} ; \xi, \eta)=C(\xi, \eta) \frac{\rho_{n-1}(z-i[\xi]-i[\eta], \bar{z}) \sigma_{n+1}(z+i[\xi]+i[\eta], \bar{z})}{\tau_{n}^{2}(z, \bar{z})}
$$

with

$$
C(\xi, \eta)=-d_{*}(\eta)\left[\frac{\kappa(\xi, \eta)}{\xi-\eta}\right]^{2}
$$

Thus we have derived the generating function for all conservation laws of all equations of the 'positive' subhierarchy,

$$
\partial_{k} \mathcal{H}_{n}^{j}=\mathcal{F}_{n}^{j k}-\mathcal{F}_{n-1}^{j k}
$$

where

$$
\mathcal{H}_{n}(\xi)=\sum_{j} \xi^{j} \mathcal{H}_{n}^{j}, \quad \mathcal{F}_{n}(\xi, \eta)=\sum_{j k} \xi^{j} \eta^{k} \mathcal{F}_{n}^{j k}
$$

Note that the constants of motion, $I_{i}$, are surely common for all equations of the ALH, but the form of the corresponding conservation law (8.18) depends upon the flow (operator $\partial_{k}$ ) with which we are dealing.

If we restrict ourselves to the zero boundary conditions, $q_{n}, r_{n} \rightarrow 0$ as $n \rightarrow \pm \infty$, then $\kappa(\xi, \eta)=\xi-\eta, d_{*}(\xi)=i \xi$ and the above formulae can be simplified to become

$$
i \partial_{1} \frac{\rho_{n-1}^{-} \sigma_{n}^{+}}{\tau_{n} \tau_{n-1}}=\frac{\rho_{n-1}^{-} \sigma_{n+1}^{+}}{\tau_{n}^{2}}-\frac{\rho_{n-2}^{-} \sigma_{n}^{+}}{\tau_{n-1}^{2}},
$$

where

$$
\omega_{n}^{ \pm}(z, \bar{z})=\omega_{n}(z \pm i[\xi], \bar{z})
$$


Considering (8.19) as an equation for power series in $\xi$ and equating coefficients of $\xi^{j}$,

$j=1,2, \ldots$, one can obtain the infinite series of conservation laws for the discrete nonlinear Schrödinger equation and the discrete modified KdV equation. The first of them is

$$
i \partial_{1} r_{n-1} q_{n}=r_{n-1} p_{n} q_{n+1}-r_{n-2} p_{n-1} q_{n}
$$

i.e. the conservation law corresponding to the constant $I_{1}$ given by (8.1).

Analogous calculations can be carried out for the 'negative' subhierarchy.

\section{$9 \quad \mathrm{ALH}$ and other hierarchies}

It has been shown in 12, 13, 14 that the ALH possesses some kind of 'universality': many integrable models can be 'embedded' into the ALH, i.e. presented as differential consequences of its equations. In this section we give a few examples of how these interrelations between the ALH and other integrable systems manifest themselves in the framework of the functional equations. We derive, starting from the functional representation of the $\mathrm{ALH}$, functional representation of some other well-known integrable equations and hierarchies.

\subsection{Functional representation of the derivative NLS hierarchy}

The functional equations for the tau-functions (4.15)-(4.17) can be presented as

$$
\begin{gathered}
q_{n}^{+}-q_{n}=\zeta \frac{\tau_{n-1}}{\tau_{n}} \frac{\sigma_{n+1}^{+}}{\tau_{n}^{+}}, \\
r_{n-1}-r_{n-1}^{+}=\zeta \frac{\rho_{n-2}}{\tau_{n-1}} \frac{\tau_{n}^{+}}{\tau_{n-1}^{+}}, \\
1+\zeta r_{n-1} q_{n}^{+}=\frac{\tau_{n}}{\tau_{n-1}} \frac{\tau_{n-1}^{+}}{\tau_{n}^{+}},
\end{gathered}
$$

where

$$
f_{n}^{ \pm}=f_{n}(z \pm i[\zeta])
$$

Shifting the arguments in the first equation, $z_{k} \rightarrow z_{k}-i \zeta^{k} / k$, and using (9.3) one can rewrite (9.1) and (9.2) as

$$
\begin{gathered}
\zeta^{-1}\left(1+\zeta q_{n} r_{n-1}^{-}\right)\left(q_{n}-q_{n}^{-}\right)=\frac{\tau_{n-1} \sigma_{n+1}}{\tau_{n}^{2}}, \\
\zeta^{-1}\left(1+\zeta q_{n}^{+} r_{n-1}\right)\left(r_{n-1}-r_{n-1}^{+}\right)=\frac{\rho_{n-2} \tau_{n}}{\tau_{n-1}^{2}} .
\end{gathered}
$$

It should be noted that the left-hand sides of these two identities, for fixed $n$, contain only two functions, $q_{n}$ and $r_{n-1}$, taken at the different values ot their arguments, while the right-hand sides do not depend on $\zeta$. Thus differentiating (9.5) and (9.6) with respect to $\zeta$, one can obtain that the quantities

$$
Q=q_{n} \quad \text { and } \quad R=r_{n-1}
$$


satisfy the system

$$
\begin{aligned}
& \frac{\mathrm{d}}{\mathrm{d} \zeta} \zeta^{-1}\left(1+\zeta Q R^{-}\right)\left(Q-Q^{-}\right)=0, \\
& \frac{\mathrm{d}}{\mathrm{d} \zeta} \zeta^{-1}\left(1+\zeta Q^{+} R\right)\left(R-R^{+}\right)=0 .
\end{aligned}
$$

In such a way we have shown that solutions of the ALH solve also (9.8) and (9.9). This closed system of functional equations can be viewed as (formal) series in $\zeta$. Expanding (9.8) and (9.9) in $\zeta$ and equating coefficients of different powers of $\zeta$ to zero one can obtain an infinite number of partial differential equations. The simplest of them are

$$
\begin{aligned}
& \partial_{2} Q-i \partial_{11} Q+2 Q R \partial_{1} Q=0, \\
& \partial_{2} R+i \partial_{11} R+2 Q R \partial_{1} R=0,
\end{aligned}
$$

which is the derivative NLS system. Others of these equations are compatible with (9.10) and (9.11) (by construction) and can be transformed to the form

$$
\begin{aligned}
\partial_{j} Q & =\mathcal{P}_{Q}(Q, R, \partial Q, \partial R, \ldots), \\
\partial_{j} R & =\mathcal{P}_{R}(Q, R, \partial Q, \partial R, \ldots),
\end{aligned}
$$

where $\partial=\partial / \partial z_{1}$, and $\mathcal{P}_{Q}$ and $\mathcal{P}_{R}$ are polynomials in $Q, R$ and their derivatives with respect to $z_{1}$. Hence one can conclude that they are nothing other but the higher equations from the hierarchy which begins with (9.10) and (9.11), i.e. the functional equations (9.8) and $(9.9)$ can be viewed as representing the derivative NLS hierarchy.

\subsection{Functional representation of the AKNS hierarchy}

To derive the functional equations corresponding to the AKNS hierarchy consider the 'four-point' (or 'two-shift') formulae (6.10). After some calculation one can obtain the identities

$$
(\zeta-\xi) \hat{Q}_{n}^{+}=\left(1+\zeta \xi \hat{Q}_{n}^{+} R_{n}\right)\left(\zeta Q_{n}^{+}-\xi \hat{Q}_{n}\right)
$$

and

$$
(\zeta-\xi) \check{R}_{n}^{-}=\left(1+\zeta \xi Q_{n} \check{R}_{n}^{-}\right)\left(\zeta R_{n}^{-}-\xi \check{R}_{n}\right)
$$

for the quantities

$$
Q_{n}=\frac{\sigma_{n+1}}{\tau_{n}} \quad \text { and } \quad R_{n}=\frac{\rho_{n-1}}{\tau_{n}} .
$$

Here

$$
f_{n}^{ \pm}=f_{n}(z \pm i[\zeta]), \quad \hat{f}_{n}=f_{n}(z+i[\xi]), \quad \check{f}_{n}=f_{n}(z-i[\xi]) .
$$

Expansion of (9.14) and (9.15) in double series in $\zeta$ and $\xi$ leads to an infinite number of partial differential equations. It can be shown that equations we need (the NLS equation and its higher analogues) appear as cofactors of the $\zeta \xi\left(\zeta^{m}-\xi^{m}\right)$-terms, while coefficients of all other terms can be expressed as their linear combinations. We not discuss further the double series representation because in our case it can be simplified and reduced to one similar to (9.8) and (9.9). Indeed, after shifting the arguments of all functions in (9.14), $z_{k} \rightarrow z_{k}-i \zeta^{k} / k-i \xi^{k} / k$, one can rewrite it in the $\xi \rightarrow 0$ limit as

$$
Q^{-}-\zeta^{2} Q^{2} R^{-}=Q-i \zeta \partial_{1} Q
$$


The right-hand side of this equation, which is of no interest for our purposes and which is linear in $\zeta$, can be eliminated by differentiating with respect to $\zeta$. This leads to the following, rather compact relation

$$
\frac{\mathrm{d}^{2}}{\mathrm{~d} \zeta^{2}}\left(Q^{-}-\zeta^{2} Q^{2} R^{-}\right)=0
$$

Analogously, equation (9.15) can be transformed to

$$
\frac{\mathrm{d}^{2}}{\mathrm{~d} \zeta^{2}}\left(R^{+}-\zeta^{2} Q^{+} R^{2}\right)=0
$$

Expanding $(9.19)$ and $(9.20)$ in power series in $\zeta$ one can subsequently obtain the NLS equation,

$$
\begin{gathered}
i \partial_{2} Q+\partial_{11} Q+2 Q^{2} R=0, \\
-i \partial_{2} R+\partial_{11} R+2 Q R^{2}=0
\end{gathered}
$$

the third-order NLS equation,

$$
\begin{gathered}
\partial_{3} Q+\partial_{111} Q+6 Q R \partial_{1} Q=0 \\
\partial_{3} R+\partial_{111} R+6 Q R \partial_{1} R=0
\end{gathered}
$$

and so on, which enables us to identify (9.19) and (9.20) with the AKNS hierarchy.

\subsection{Functional representation of the Davey-Stewartson hierarchy}

In this paper (as well as in the paper [1]) we discussed the 'positive' and 'negative' subhierarchies separately. At the same time it is already known that the combined action of both types of the ALH flows, $\partial / \partial z_{j}$ and $\partial / \partial \bar{z}_{k}$, leads to some interesting equations, first of all the Davey-Stewartson system (see [14]) and the 2D Toda lattice (see [13]). Now we demonstrate some consequences of the functional equations related to both subhierarchies considered together.

Equations (4.15) and (4.16),

$$
\begin{aligned}
& q_{n}-q_{n}^{-}=\zeta \frac{\tau_{n-1}^{-} \sigma_{n+1}}{\tau_{n}^{-} \tau_{n}}, \\
& r_{n}-r_{n}^{+}=\zeta \frac{\rho_{n-1} \tau_{n+1}^{+}}{\tau_{n} \tau_{n}^{+}}
\end{aligned}
$$

where the designation

$$
f_{n}^{ \pm}=f_{n}(z \pm i[\zeta], \bar{z})
$$

is used, can be rewritten, by means of (4.17), as

$$
\begin{gathered}
q_{n}^{-}-\zeta^{2} q_{n} Q_{n} R_{n}^{-}=q_{n}-\zeta \frac{\tau_{n-1} \sigma_{n+1}}{\tau_{n}^{2}}, \\
r_{n}^{+}-\zeta^{2} r_{n} Q_{n}^{+} R_{n}=r_{n}-\zeta \frac{\rho_{n-1} \tau_{n+1}}{\tau_{n}^{2}}
\end{gathered}
$$


with $Q_{n}$ and $R_{n}$ being defined by (9.16). As in the examples above the right-hand side of these relations can be eliminated with the help of the $\mathrm{d} / \mathrm{d} \zeta$ operator. To obtain a closed system it remains to express the quantity $Q_{n} R_{n}^{-}$in terms of $q_{n}$ and $r_{n}$. This can be done using the 'negative' functional equations for the tau-functions. Indeed, from (4.24), (4.25) and (4.20), after some calculations omitted here one can derive the following relation:

$$
-i \bar{\partial}\left(Q R^{-}\right)=\zeta^{-1}\left(q r-q^{-} r^{-}\right), \quad \bar{\partial}=\partial / \partial \bar{z}_{1}
$$

or

$$
Q R^{-}=i \zeta^{-1} \bar{\partial}^{-1}\left(q r-q^{-} r^{-}\right)
$$

which leads to

$$
\frac{\mathrm{d}^{2}}{\mathrm{~d} \zeta^{2}}\left[q^{-}+i \zeta q \bar{\partial}^{-1}\left(q^{-} r^{-}-q r\right)\right]=0
$$

and

$$
\frac{\mathrm{d}^{2}}{\mathrm{~d} \zeta^{2}}\left[r^{+}+i \zeta r \bar{\partial}^{-1}\left(q r-q^{+} r^{+}\right)\right]=0 .
$$

The lowest-order partial differential equations corresponding to this system, i.e. the terms proportional to $\zeta^{2}$ in square brackets, can be presented as

$$
\begin{gathered}
i \partial_{2} q+\partial_{11} q-2 C q=0, \\
-i \partial_{2} r+\partial_{11} r-2 C r=0, \\
\bar{\partial} C=\partial q r .
\end{gathered}
$$

It is not difficult to show that these equations, which describe an integrable system proposed by Zakharov [15], together with their 'negative' counterparts are equivalent to the Davey-Stewartson system (see [14]). Thus one can consider (9.32) and (9.33) as functional equations representing the Davey-Stewartson hierarchy. Similar representation has been derived earlier by Bogdanov and Konopelchenko using the analytic-bilinear approach [16].

\section{Conclusion}

The ALH is usually considered as a set of differential-difference equations. However, the differential 'component' of the hierarchy can be viewed as a consequence of the difference one. It is clearly seen in the framework of the inverse scattering transform. If one fixes the $U$-matrix in (2.1) and restricts oneself to the polynomial $V$-matrices, one almost completely determines the latter, i.e. all differential equations of the hierarchy are 'hidden' in the difference problem (2.1). Thus it is possible to obtain a wide range of results related to the hierarchy without dealing with separate flows. One of the main aims of this paper was to embody this idea (Kyoto approach [17]). The functional equations are a useful tool for such 'global' consideration: an infinite set of differential equations (continuous flows) is replaced with one equation (discrete flow). Another motive behind this work was the following one. The ALH has a rather long history. Much effort has been spent to study the discrete NLS and discrete modified KdV equations, the simplest equations of the ALH. However, the general, abstract, theory of this hierarchy (say, similar to the well-elaborated KP theory) has not yet been developed. The results presented above 
seem to give a perspective from this viewpoint. For example the representation (2.29) can be a starting point to investigate the group-algebraic structures behind the ALH. The appearance of Miwa's shifts in the main equations of this paper, together with the Pluckker-like relations 6.12, indicates that we are close to the description of the ALH using the language of the Grassmannians and it is interesting to continue the studies in this direction, to introduce vertex operators, to construct the free-field realization of the ALH and to address other questions which are of much interest in the modern theory of the integrable systems.

\section{Acknowledgements}

This work was partly carried out during the author's stay at the Abdus Salam International Centre for Theoretical Physics which is gratefully acknowledged for its kind hospitality and has been supported by the Ministerio de Ciencia, Ciltura y Deporte of Spain under grant SB1999-AH777133. I am also grateful to the referee of this paper for careful reading the manuscript and making a few crucial remarks.

\section{References}

[1] Vekslerchik V E, Functional Representation of the Ablowitz-Ladik Hierarchy, J. Phys. A: Math. Gen. 31 (1998), 1087-1099.

[2] Ablowitz M J and Ladik J F, Nonlinear Differential-Difference Equations, J. Math. Phys. 16 (1975), 598-603.

[3] Miller P D, Ercolani N M, Krichever I M and Levermore C D, Finite Genus Solutions to the Ablowitz-Ladik Equations, Comm. Pure Appl. Math. 48 (1996), 1369-1440.

[4] Suris Yu B, A Note on an Integrable Discretization of the Nonlinear Schrödinger Equation, Inverse Problems 13 (1997), 1121-1136.

[5] Newell A C, Solitons in Mathematics and Physics, SIAM, Philadelphia, 1985.

[6] Miwa T, On Hirota's Difference Equations, Proc. Jap. Acad., Ser. A 58 (1982), 9-12.

[7] Ablowitz M J and Ladik J F, A Nonlinear Difference Scheme and Inverse Scattering, Stud. Appl. Math. 55 (1976), 213-229.

[8] Suris Yu B, On an Integrable Discretization of the Modified Korteweg-de Vries Equation, Phys. Lett. A 234 (1997), 91-102.

[9] Vekslerchik V E, Finite Genus Solutions for the Ablowitz-Ladik Hierarchy, J. Phys. A: Math. Gen. 32 (1999), 4983-4994.

[10] Ablowitz M J and Ladik J F, Nonlinear Differential-Difference Equations and Fourier Analysis, J. Math. Phys. 17 (1976), 1011-1018.

[11] Ablowitz M J and Segur H, Solitons and the Inverse Scattering Transform, SIAM, Philadelphia, 1981.

[12] Vekslerchik V E, An $O(3,1)$ Nonlinear $\sigma$-Model and the Ablowitz-Ladik Hierarchy, J. Phys. A: Math. Gen. 27 (1994), 6299-6313. 
[13] Vekslerchik V E, The 2D Toda Lattice and the Ablowitz-Ladik Hierarchy, Inverse Problems 11 (1995), 463-479.

[14] Vekslerchik V E, The Davey-Stewartson Equation and the Ablowitz-Ladik Hierarchy, Inverse Problems 12 (1996), 1057-1074.

[15] Zakharov V E, The Inverse Scattering Method, in Solitons, Editors: R K Bullough and P J Caudrey, Springer-Verlag, New York, 1980, 243-285.

[16] Bogdanov L V and Konopelchenko B G, Analytic-Bilinear Approach to Integrable Hierarchies. II. Multicomponent KP and 2D Toda Lattice Hierarchies, J. Math. Phys. 39 (1998), 47014728.

[17] Jimbo M and Miwa T, Solitons and Infinite Dimensional Lie Algebras, Publ. Res. Inst. Math. Sci. 19 (1983), 943-1001. 